\title{
The Effects of External Jugular Compression Applied during High Intensity Power, Strength and Postural Control Tasks
}

\author{
Christopher A. DiCesare, MS ${ }^{1}$ Kim D. Barber Foss, MS, ATC ${ }^{1,2}$ Staci Thomas, MS ${ }^{1}$ \\ Daniel K. Schneider, BS ${ }^{1,3}$ Nicholas M. Edwards, MD, MPH ${ }^{4}$ Gregory D. Myer, PhD ${ }^{1,3,5,6,7}$
}

\footnotetext{
${ }^{1}$ The SPORT Center and Human Performance Laboratory, Division of Sports Medicine, Cincinnati Children's Hospital Medical Center, Cincinnati, Ohio

2 Rocky Mountain University of Health Professions, Provo, Utah

${ }^{3}$ College of Medicine, University of Cincinnati, Cincinnati, Ohio

${ }^{4}$ Department of Orthopaedics, University of Minnesota,

Minneapolis, Minnesota

${ }^{5}$ Department of Pediatrics, University of Cincinnati, Cincinnati, Ohio

${ }^{6}$ Department of Orthopaedics, University of Pennsylvania,

Philadelphia, Pennsylvania

7 The Micheli Center for Sports Injury Prevention, Boston's Children's Hospital, Waltham, Massachusetts
}

Curr Res Concussion 2017;4:e23-e31.

\author{
Address for correspondence Christopher A. DiCesare, MS, \\ Division of Sports Medicine, Cincinnati Children's Hospital, \\ 3333 Burnet Avenue, MLC-10001, Cincinnati, OH 45229 \\ (e-mail: christopher.dicesare@cchmc.org).
}

\begin{abstract}
Keywords

- concussion

- jugular vein

- biomechanics

Introduction Current strategies focused on mitigating concussion in sport have demonstrated limited effectiveness. There is a paucity of research on the optimization of intracranial brain dynamics to mitigate concussion; in the present study, we investigate a novel device that provides mild jugular vein compression and may provide adjunctive protection to protect the brain internally from concussive and sub-concussive impacts. The purpose of this study was to assess the tolerance and acceptance of this device in a population of normal, healthy adults undergoing exertion similar to that is experienced while participating in sports-related competition, while monitoring changes in their biomechanical, strength, power, and postural stability capabilities.

Material and Methods A total 18 participants (8 females, 10 males) were tested and included in the final analysis. Laboratory data collection consisted of two separate testing sessions for all participants. During the first testing session, participants were tested while wearing either the mild jugular vein compression neck device or a sham arm device (Sham) that was worn on the upper arm and did not alter venous return; participants exchanged devices for the second session. Participants underwent a battery of physical and neurological tests that included three-dimensional (3-D) biomechanical analysis, dynamic postural control testing using a stabilometer, isokinetic strength testing using a dynamometer, and a maximum vertical jump test. Results Evaluation of vital biomechanics, postural control and dynamic stabilization, isokinetic strength, and power in this population showed no statistically significant
\end{abstract}

received

December 7, 2016 accepted after revision July 31,2017
DOI https://doi.org/

10.1055/s-0037-1606580. ISSN 2368-0539.
Copyright $\odot 2017$ by Thieme Medical Publishers, Inc., 333 Seventh Avenue, New York, NY 10001, USA. Tel: +1(212) 584-4662.
License terms

(c) (1) $\ominus$ (\$) 
effect of wearing a mild jugular vein compression neck device compared with a Sham armband.

Conclusions The data evaluated in the current project indicate that the device is safe during high intensity and dynamic postural stabilization exercise and does not alter normal physical or neuromuscular capabilities during physical activity.

Current efforts focused on mitigating concussion in sport have demonstrated limited effectiveness. Many strategies employed to improve the risk of closed head injury in sport have focused on impact dispersion devices, e.g., helmets, and not on the concussion mechanism itself-the movement of the brain within the cranium-a phenomenon otherwise known as slosh. Recent evidence has suggested that a potential strategy to mitigate the effects of head impacts may be through the modulation of intracranial blood volume through jugular vein impedance. ${ }^{1-4}$ Mild compression of the jugular veins slows jugular outflow and results in an increase in the volume of the venous capacitance vessels of the cranium (Fisher JA, Duffin J, Mikulis D, Sobczyk O. The effect of jugular vein compression on cerebral hemodynamics in healthy subjects. Unpublished Report. 2013), filling the cranium's compensatory reserve volume. This reduction in jugular outflow and the subsequent increase in intracranial volume has been suggested to increase the stiffness of the brain, ${ }^{5}$ making it less likely to be distorted upon experiencing shear and rotary force, and it ultimately results in a cradling effect that reduces movement of the brain within the cranium, or slosh. This mechanism for protecting the brain is mimicked from a naturally occurring system found in highly g-force tolerant creatures in the animal kingdom utilizing the omohyoid muscle.

Subsequently, a novel jugular vein compression collar device has been developed to modulate intracranial blood volume via jugular outflow diversion. The landmark evaluation of this device in rats using the Marmarou protocol, which imparted a $900 \times \mathrm{g}$ force model into their crania, showed an $83 \%$ reduction in resultant amyloid precursor proteins (APP, a signature axonal injury indicator). ${ }^{1}$ Further, the investigators showed a statistically significant improvement in tensor functional (MRI) findings of microstructural injury (and functional electroencephalogram [EEG] alterations) in collared verses non-collared high school hockey players. ${ }^{3}$ Most recently, a similar statistically significant protective effect in American high school football players wearing the collar when looking at tensor MRI and correlated the findings to functional MRI (fMRI) working memory improvement. ${ }^{5}$ Despite the apparent effectiveness of this device in preserving brain structure against the effects of concussive and sub-concussive impacts, the tolerance and safety of the device have not been established, particularly which concerns the physical and neuromuscular capabilities that dictate sport performance.

In the present study, we examine a device that provides mild jugular vein compression that may provide a valuable adjunctive mechanism to protect the brain internally from concussive and sub-concussive impacts. The purpose of this study was to assess the tolerance and acceptance of this device in a population of normal, healthy adults undergoing exertion similar to that is experienced while participating in sports-related competition, while monitoring changes in their biomechanical, strength, power, and postural stability capabilities.

\section{Methods}

\section{Participants}

- Table 1 lists the inclusion and exclusion criteria for participation in the current study. A total of 20 subjects

Table 1 Inclusion and exclusion criteria for participation in the present study

\begin{tabular}{|c|c|}
\hline Inclusion criteria & Exclusion criteria \\
\hline $\begin{array}{l}\geq 18 \text { years of age } \\
\text { Able to tolerate hypercapnia for } 1-2 \text { minutes } \\
\text { Of normal health }\end{array}$ & $\begin{array}{l}\text { Unable to provide written consent } \\
\text { History of neurological deficits, previous cerebral infarction, or head trauma } \\
\text { Recent penetrating brain trauma (within } 6 \text { months) } \\
\text { Medical contraindications to hypercapnia or restriction of venous outflow via the } \\
\text { internal jugular veins (known increased intracerebral pressure, metabolic } \\
\text { acidosis, or alkalosis) } \\
\text { Glaucoma (narrow angle or normal tension) } \\
\text { Hydrocephalus } \\
\text { Known carotid hypersensitivity or increased intracranial pressure } \\
\text { Central vein thrombosis } \\
\text { Known open eye injuries, neck injuries, or airway obstructions } \\
\text { Known seizure disorder } \\
\text { Known cardiovascular or respiratory issues } \\
\text { Known untreated hypertension, cardiomyopathy, or exercise intolerance } \\
\text { Any indication of cardiovascular risk by the PAR-Q } \\
\text { Recent lower extremity injury (within } 6 \text { months) } \\
\text { Recent ACL reconstruction (within } 2 \text { years) } \\
\text { Any altered level of consciousness }\end{array}$ \\
\hline
\end{tabular}

Abbreviations: ACL, anterior cruciate ligament; PAR-Q, Physical Activity Readiness Questionnaire. 
(10 males, 10 females) volunteered for participation in this study. All recruited subjects met the inclusion criteria and were allowed to undergo testing. On one occasion following testing, a female participant was contraindicated with pretest glucose measures exceeding $367 \mathrm{mg} / \mathrm{dL}$. Another female participant indicated that she felt dizzy following the maximum oxygen $\left(\mathrm{O}_{2}\right)$ uptake testing and did not feel that she could complete the vestibular testing. She was stopped from further testing until she felt fully recovered. Both participants were able to complete the testing; however, the study team concluded a priori to statistical analyses that these factors met exclusion criteria and did not include these participants in the final analyses. A total of eight females (mean height $1.64 \pm 0.05 \mathrm{~m}$; weight $68.8 \pm 19.0 \mathrm{~kg}$; and age $22.4+1.2$ years) and 10 males (mean height $1.76 \pm 0.06 \mathrm{~m}$; weight $82.5 \pm 12.4 \mathrm{~kg}$; and age $23.8 \pm 1.9$ years) study participants were tested and included in the final analysis. Study protocol was approved by the Cincinnati Children's Hospital Institutional Review Board, and informed written consent was obtained from each participant prior to testing.

\section{Data Collection}

This study utilized a randomized, crossover study design. Laboratory data collection consisted of two separate testing sessions for all participants. During the first testing session, participants were tested while wearing either the mild jugular vein compression device (Collar) or a sham arm device (Sham) that was worn on the upper arm and did not alter venous return; during the second session, participants who wore the neck device for the first session were tested wearing the sham arm device, and vice versa. The order of these testing sessions was randomized by the study coordinator at the time of study enrollment and prior to the first testing session.

\section{Device Description and Application}

The Collar was designed to apply a gentle compressive force in order to cause a slight occlusion of the internal jugular veins, and the Sham was an elastic arm sweatband intended to be worn on the upper arm. The Collar incorporated two bulges that bilaterally compressed the regions of the neck superficial to the internal jugular veins. Participants were outfitted with either the Collar or the Sham at the start of each testing session by a staff member appropriately trained in fitting each device to the proper location. To ensure proper fitting and placement, an ultrasound was performed upon fitting to examine the immediate effect of device placement on venous return in either the neck or arm, confirming either the presence of alteration while wearing the Collar or absence of alteration while wearing the Sham (-Fig. 1). Ultrasound frequency was set at 6 to $12 \mathrm{MHz}$, and the predicted exposure time was 5 minutes per participant. Following the testing session in which the Collar was worn, participants were reexamined to verify that blood flow within the jugular veins was reduced, and flow within the carotid arteries and all portions of the cerebrum was preserved.

\section{Three-Dimensional Motion Analysis}

A 10-camera, real-time, high speed, passive optical 3-D motion analysis system (Raptor-E, Motion Analysis Corp., Santa Rosa, CA) was used to collect motion data at $240 \mathrm{~Hz}$. One investigator instrumented each participant with 43 retroreflective markers (9-mm diameter) placed on the sacrum, thorax (posterior cluster of three non-collinear markers), bilaterally on the shoulder, upper arm, elbow, wrist, anterior superior iliac spine, greater trochanter, mid-thigh, medial and lateral femoral condyles, tibial tubercle, mid-shank, distal shank, medial and lateral malleoli, heel, dorsal surface of the midfoot, lateral foot (fifth metatarsal), and toe (between the second and third metatarsal). The four-foot markers were embedded in a

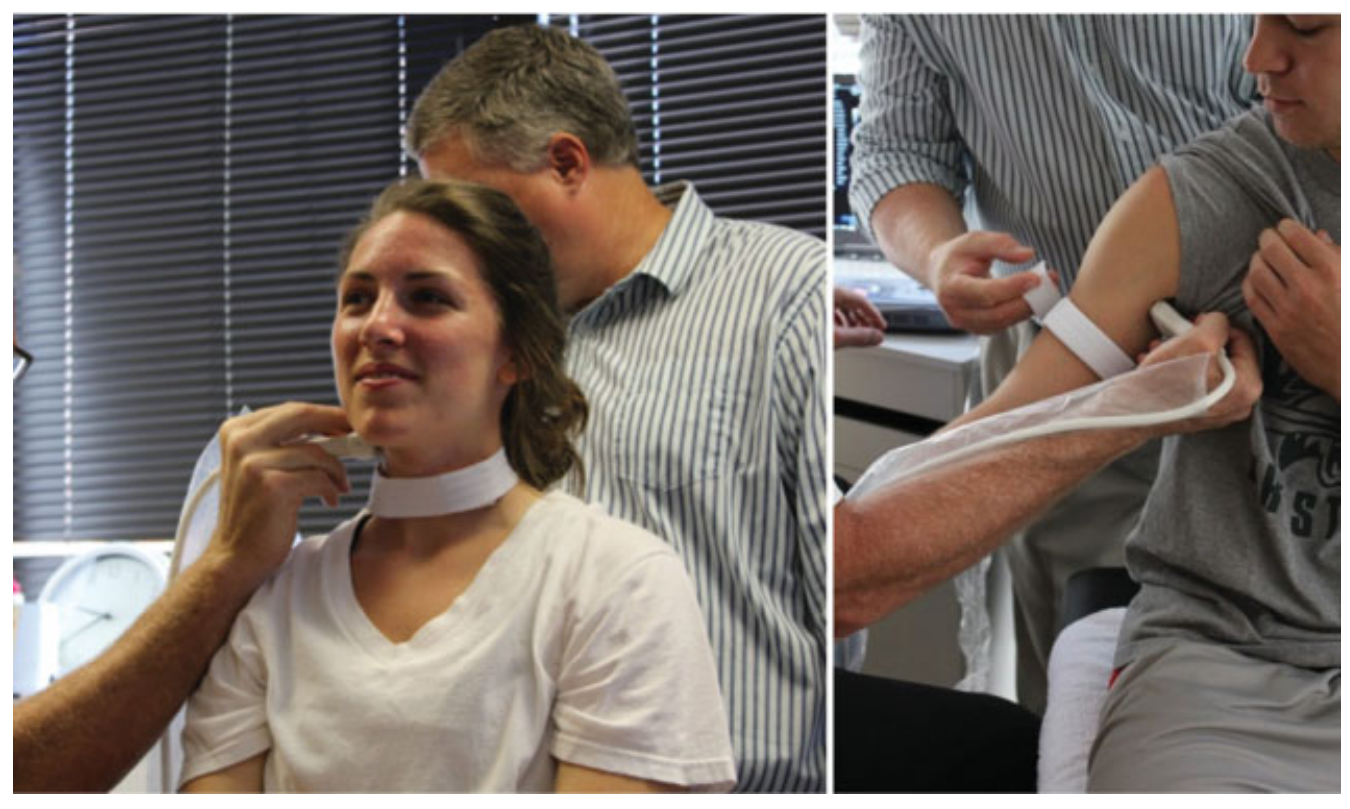

Fig. 1 Ultrasound being performed on a participant with the Collar device (left) and Sham device (right). 
standardized running show (Supernova Glide 2, Adidas, Herzogenaurach, Germany). The motion analysis system was calibrated according to the manufacturer's recommendations. Force data from two embedded force platforms (AMTI, Watertown, MA) were collected at $1200 \mathrm{~Hz}$ and were synchronized with motion data.

A standing static trial was collected in which the participant was instructed to stand in a neutral position with foot placement standardized to the laboratory's global coordinate system. The participants then performed three-drop vertical jump trials from a 31-cm box (-Fig. 2). To perform the drop vertical jump, participants dropped forward off of the box and landed on the force platforms. Immediately upon landing, the participants performed a maximum effort vertical jump while reaching up with both hands with the goal of grasping an overhead target. Three-dimensional kinematic and kinetic measures were then calculated in Visual3D (CMotion, Germantown, MD) from the motion and force data. Peak values were recorded during the landing phase of stance, defined as the period from the initial contact with the force plate to the point at which the body's center of mass reached its minimum vertical displacement.

\section{Dynamic Postural Control and Stability}

Dynamic, unilateral postural control was measured using a stabilometer (Biodex Medical Systems, Inc., Shirley, NY).

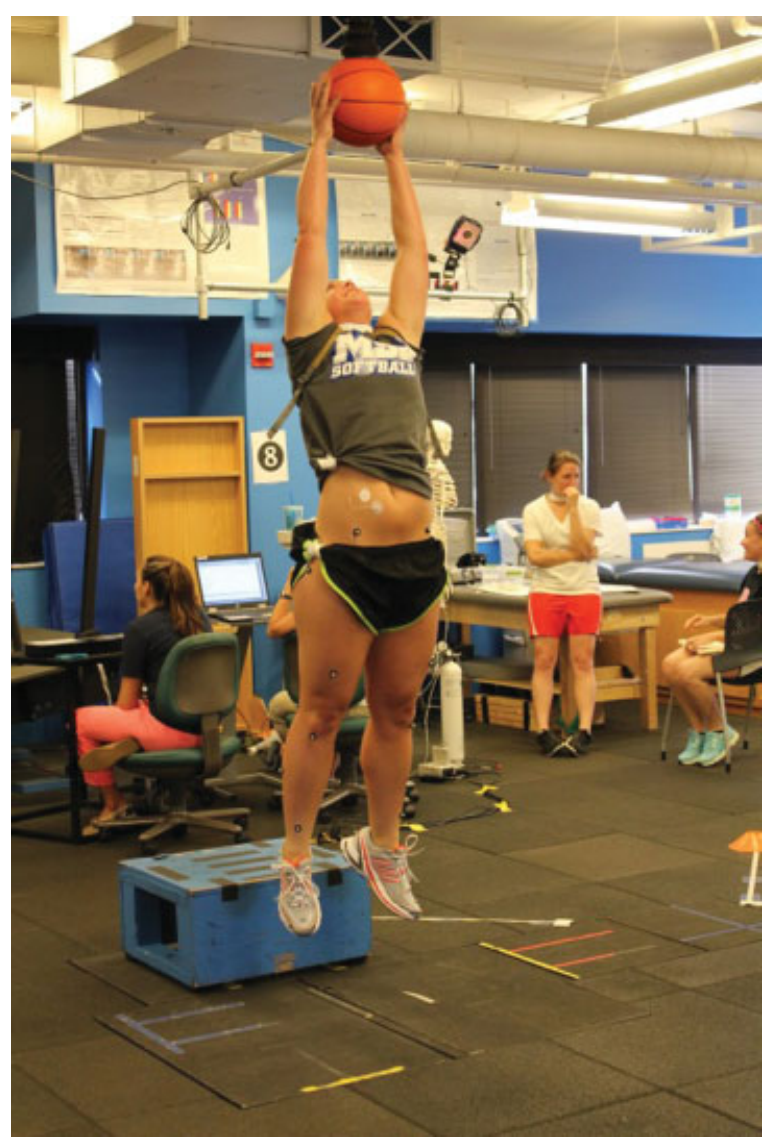

Fig. 2 The drop vertical jump task from which kinematic and kinetic measures were calculated during 3-D motion data capture. 3-D, three dimensional.
Participants were instructed to stand on one foot with the knee slightly flexed, and the other knee flexed to $90^{\circ}$ on the free-moving stability platform for 20 seconds (-Fig. 3). Each leg was tested three times, and the average of the three scores was recorded. Dynamic postural control was analyzed by comparing actual scores and standard deviations reported by the stabilometer with each device to determine the effect of wearing each device on each participant's proprioception and coordination.

Participants also completed the Y-Balance test, which is a unilateral assessment of dynamic stability during which the subject balances on one limb and performs a maximal reach with the opposite limb in the anterior, posteromedial, and posterolateral directions (-Fig. 4). Prior to completion of the test, athletes were given explicit instructions and the opportunity to perform several practice trials.

\section{Isokinetic Strength and Power}

Knee flexor and extensor strength was measured using an isokinetic dynamometer (Biodex Medical Systems, Inc., Shirley, NY). Participants were seated on the dynamometer with their trunk perpendicular to the floor, their hip flexed to $90^{\circ}$, and their knee flexed to $90^{\circ}$ (-Fig. 5). The athlete was instructed to push and pull against a padded buttress and a strap positioned just above the ankle by flexing and extending at the knee. Immobilization consisted of a thoracic strap, a

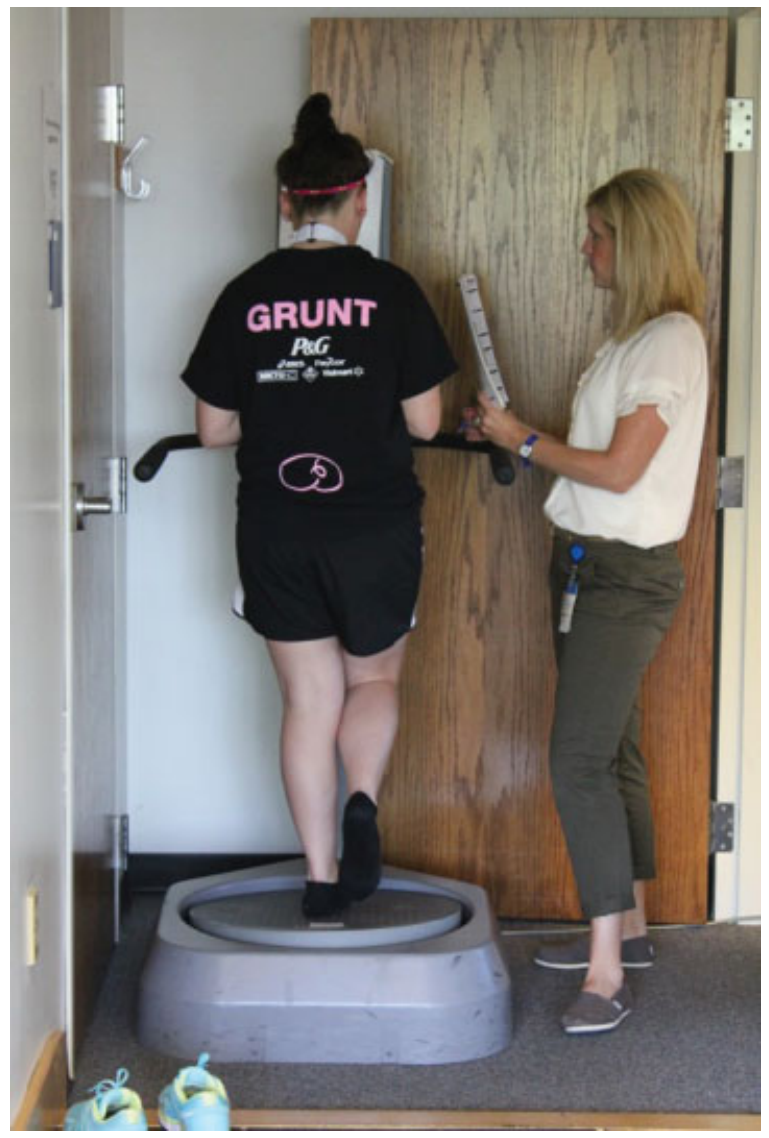

Fig. 3 Assessment of dynamic unilateral postural stability task using a stabilometer from which proprioception and coordination measures were calculated. 


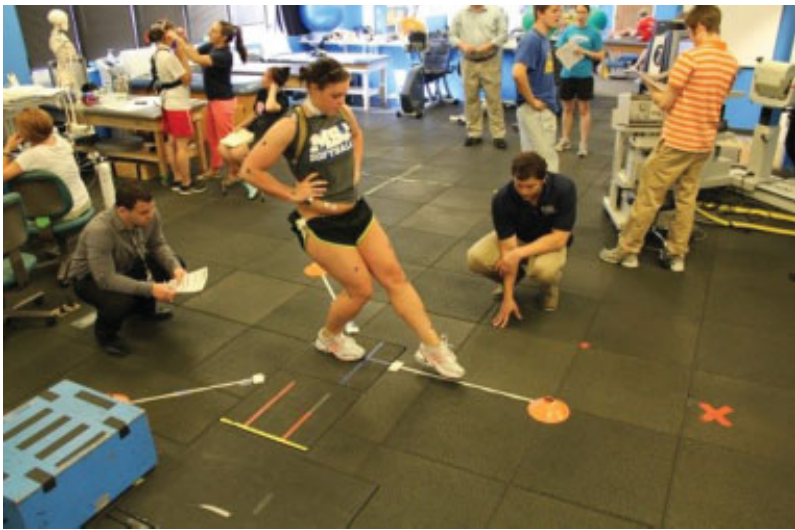

Fig. 4 Assessment of dynamic stability while performing the unilateral Y-Balance test.

waist strap, bilateral thigh straps, and a shin strap. The test was completed for each limb with at an angular velocity of $300^{\circ} \%$ second for a total of 10 repetitions. Peak flexion and extension torques were recorded. Participants also completed a maximum vertical jump test. Participants were instructed to perform a standing maximum vertical jump to reach an overhead target for a total of three trials. The maximum jump height over the three trials was recorded.

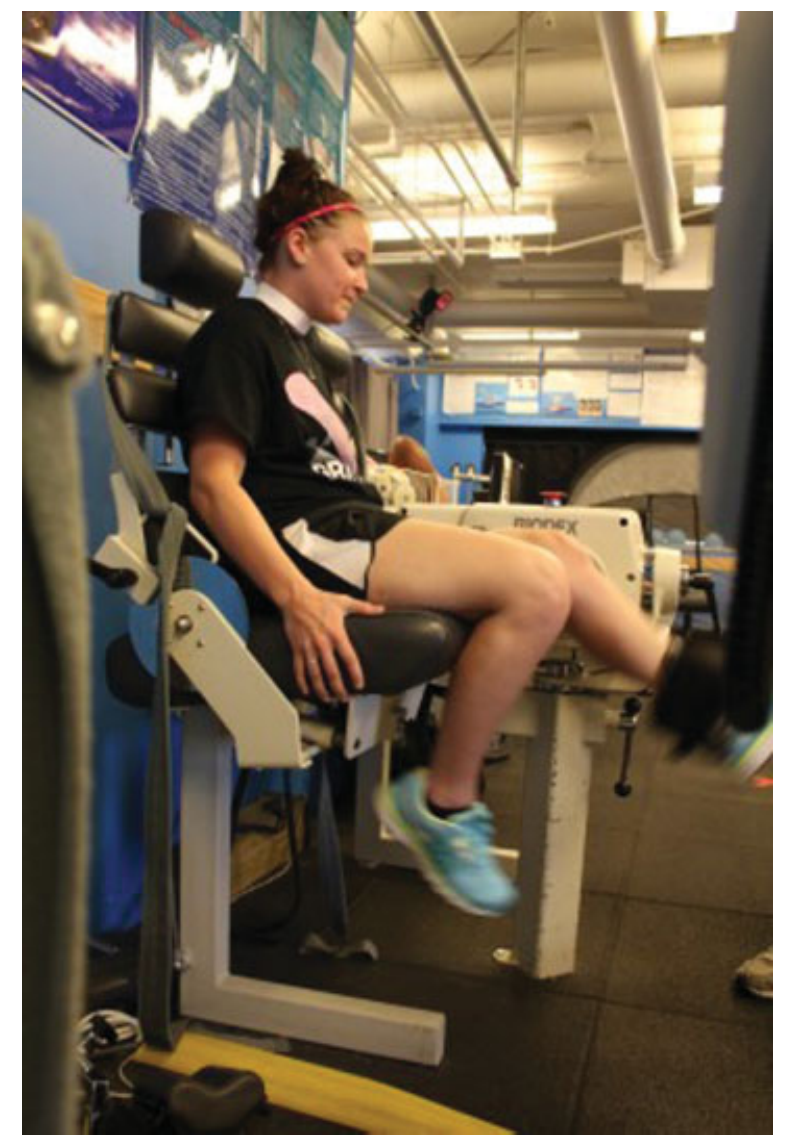

Fig. 5 Assessment of knee extensor strength using an isokinetic dynamometer.

\section{Statistical Analysis}

All statistical analyses were performed using SPSS statistical software (SPSS Inc., Chicago, IL). Descriptive information was calculated for each variable of interest and was compared between testing conditions (Sham versus Collar) using paired $t$-tests assuming equal variance. Statistical significance was established $a$ priori at $p<0.05$. Bland-Altman plots ( $95 \%$ confidence intervals) were employed to evaluate each variable to determine systematic shift between the mean Sham condition and the Collar condition to verify if there were any associations between the differences of the two measures and their means.

\section{Results}

Evaluation of monitored kinematic measurements showed no significant effect of wearing the Collar compared with Sham (all $p>0.05$ ) ( - Fig. 6, - Table 2). Similar results were found in the kinetic analysis (-Fig. 7, - Table 3); there was a significant reduction in low magnitude in knee abduction moment found in the group as they wore the Collar. Additionally, evaluation of monitored dynamic postural control and stabilization measurements showed no significant effect of wearing the device compared with the Sham (all $p>0.05$ ) ( $\mathbf{- T a b l e s} \mathbf{4}$ and $\mathbf{5}$ ). For the monitored isokinetic strength and power (vertical jump) measurements, evaluation also showed no significant effect of wearing the Collar compared with the Sham (all $p>0.05$ ) (-Table 6).

\section{Discussion}

A natural concern about jugular vein compression and the backfilling of venous blood into the compensatory reserve volume is whether or not it could affect the athlete's ability to compete at his or her highest level. The purpose of the present study was to monitor changes in biomechanical, strength, power, and postural stability capabilities in normal, healthy adults undergoing sub-maximal exertion while wearing a device that provided mild jugular vein compression to identify any detrimental actions that could adversely affect an athlete's athletic performance goals and abilities.

The physical and neuromuscular capabilities that were measured in this study were unchanged as a result of wearing the Collar. Cumulatively, the data evaluated in the current project indicate that the device evaluated in normal athletes is safe during strenuous, yet sub-maximal exercise and does not alter normal physical and neurologic capabilities during physical activity. Specifically, all facets of the study were geared to correlate to the activities one might be exposed to during any normal field of play, and the physiologies tested were chosen as reflective of the peak potentials of many objectively identified parameters. Although carbon dioxide $\left(\mathrm{CO}_{2}\right)$ reactivity has shown no anatomical alterations in blood flow and $\mathrm{O}_{2}$ uptake, even at the molecular level, functionally, every attempt was made in this effort to demonstrate any decline in all the physiological capabilities under the scrutiny of a state-of-the-art human performance laboratory. 

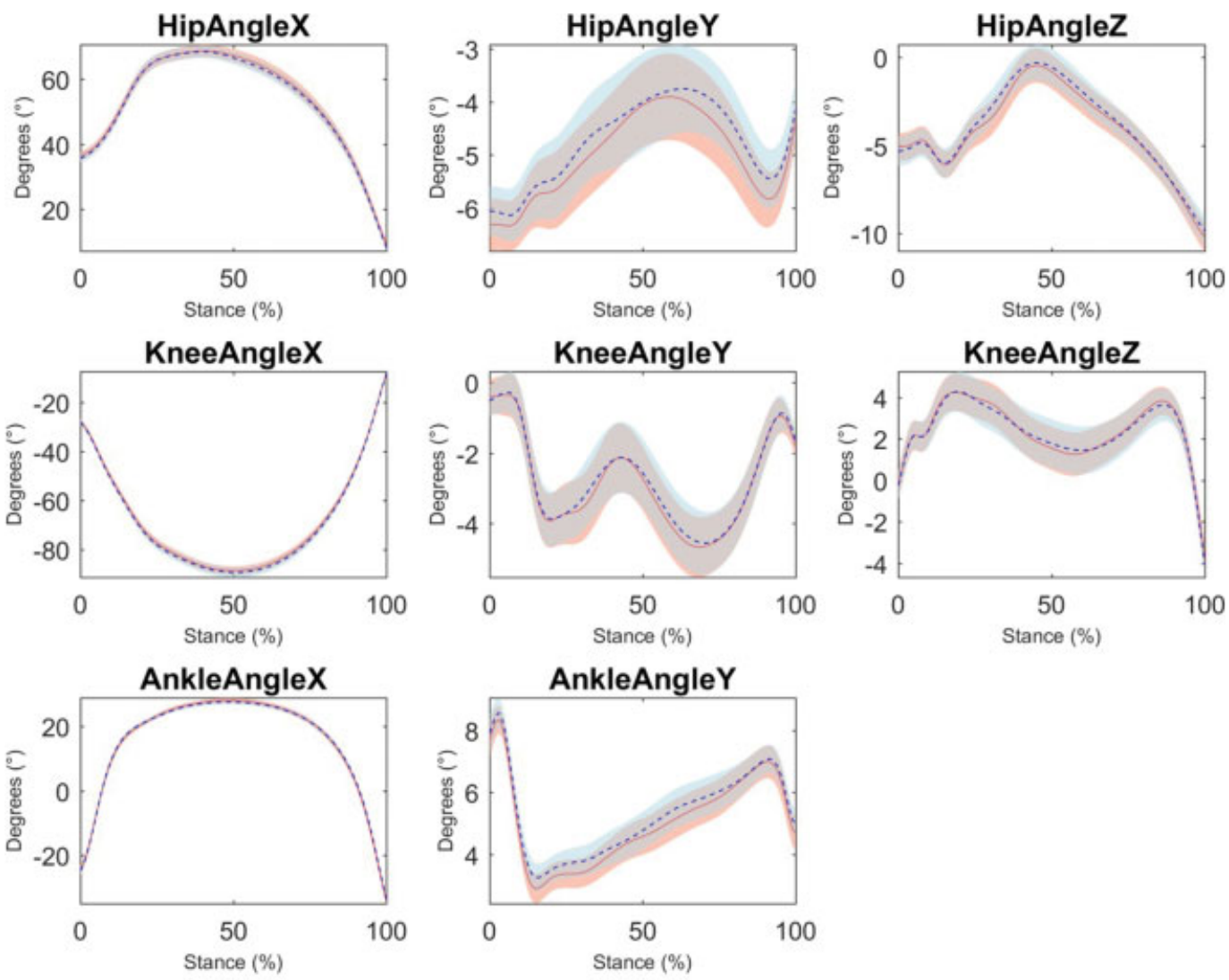

Fig. $6 \mathrm{Hip}$, knee, and ankle kinematic time series in the sagittal (left column), frontal (middle column), and transverse (right column) planes during the drop vertical jump task for subjects wearing the Sham device (blue) versus the Collar device (red).

From an evolutionary standpoint, it would be reasonable to conclude that phenotypic presence of the omohyoid and digastric compression of the jugular vein would not be selected for if their presence had a deleterious effect on survival.

To be successful while playing sports involving high frequency head impacts, such as American football, soccer, ice hockey, and lacrosse, athletes typically need to possess certain qualities-namely, reactive change of direction abilities accomplished via pivoting, cutting, and jumping.
Biomechanical asymmetries identified via the testing protocols utilized in the present study have been associated with increased injury risk during sports participation. ${ }^{6}$ For example, leg dominance, or unbalanced strength and dynamic coordination in the two lower extremities, ${ }^{7,8}$ is associated with increased risk of anterior cruciate ligament (ACL) injury in athletes participating in sports. ${ }^{9,10}$ Imbalances in muscular strength and dynamic postural control also contribute to injury risk in athletes. ${ }^{10-13}$ The current findings did not reveal any differences in strength or

Table 2 Comparison of mean peak lower extremity kinematic variables during the drop vertical jump task between the Sham and Collar conditions

\begin{tabular}{|l|l|l|l|l|l|}
\hline \multirow{2}{*}{ Measure $\left(^{\circ}\right.$ ) } & \multicolumn{2}{|l|}{ Sham } & Collar & \multirow{2}{*}{$p$} \\
\cline { 2 - 5 } & Mean & SD & Mean & SD & \\
\hline Hip flexion & 69.41 & 12.20 & 69.73 & 12.16 & 0.830 \\
\hline Hip abduction & 2.59 & 3.26 & 3.12 & 2.88 & 0.128 \\
\hline Hip internal rotation & 1.55 & 4.78 & 0.95 & 4.32 & 0.295 \\
\hline Knee flexion & 89.03 & 13.18 & -88.36 & 11.19 & 0.551 \\
\hline Knee abduction & 6.14 & 4.84 & 5.82 & 4.55 & 0.575 \\
\hline Knee internal rotation & 6.51 & 5.05 & 6.38 & 4.52 & 0.799 \\
\hline Ankle dorsiflexion & 27.76 & 4.47 & 28.24 & 4.79 & 0.210 \\
\hline Ankle eversion & 1.68 & 2.63 & 1.53 & 2.38 & 0.624 \\
\hline
\end{tabular}

Abbreviation: SD, standard deviation. 

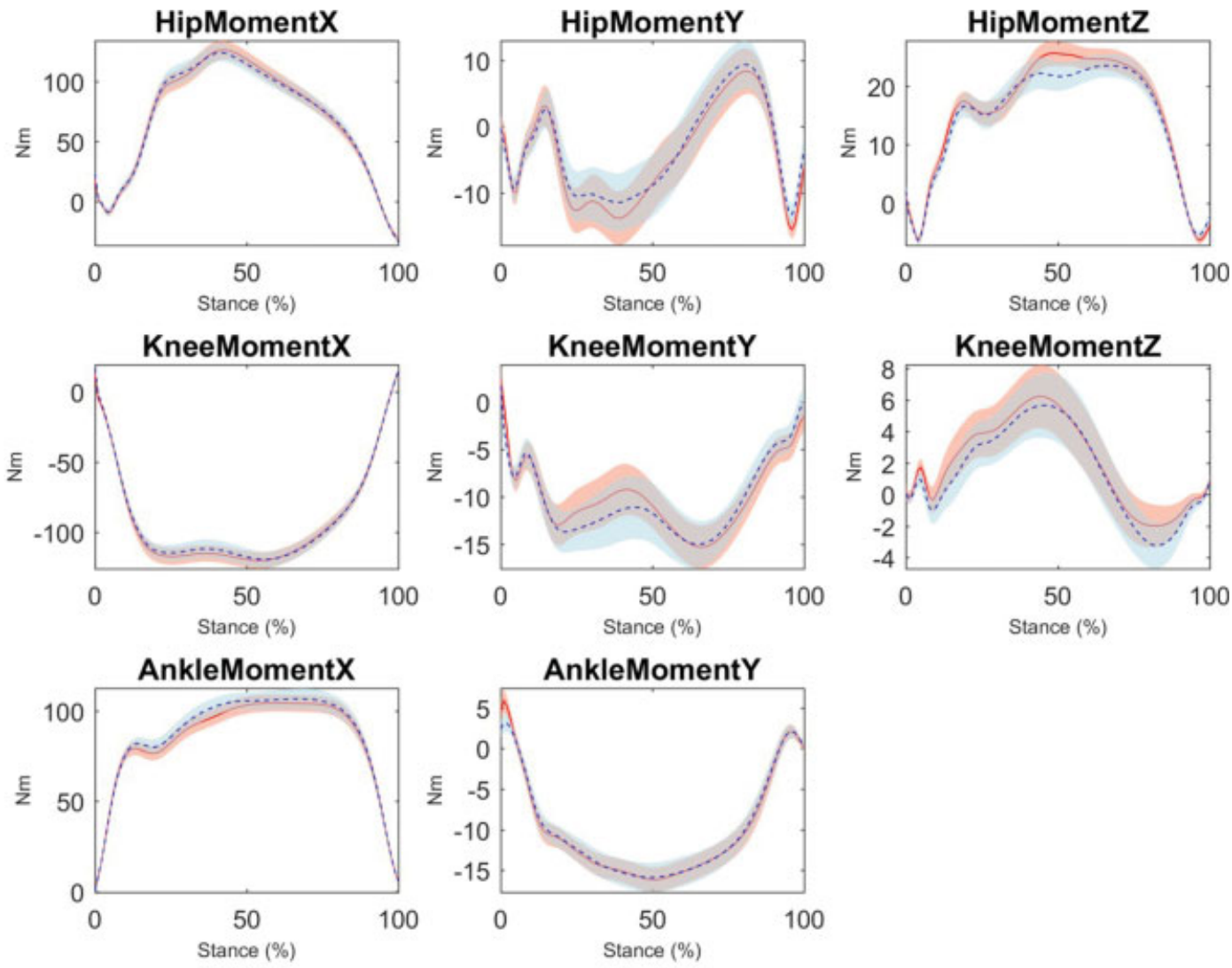

Fig. $7 \mathrm{Hip}$, knee and ankle kinetic time series in the sagittal (left column), frontal (middle column), and transverse (right column) planes during the drop vertical jump task for subjects wearing the Sham device (blue) versus the Collar device (red).

postural control suggesting that wearing a device that mitigates mild jugular vein compression does not pose additional risk of lower extremity injury. The complex neuromuscular coordination required to safely participate in high-risk sports movements were unaffected by the addition of the Collar in the present study as demonstrated by similar movement mechanics measured during the maximal effort drop vertical jump task. Of the aforementioned neuromuscular characteristics, the only difference observed during testing was a slightly decreased knee abduction moment in the Collar group relative to that in the Sham group. As increased knee abduction moments are associated with increased risk of ACL injury, ${ }^{6,14}$ this finding may be considered negligible, if not marginally beneficial in the athletic population for whom the device is designed. As the Collar did not significantly affect the multitude of other neuromuscular characteristics evaluated during the testing, the light compression provided by the device around the neck and the subsequent venous sinus engorgement likely does not negatively modify blood flow to the

Table 3 Comparison of mean peak lower extremity kinetic variables during the drop vertical jump task between the Sham and Collar conditions

\begin{tabular}{|l|l|l|l|l|l|}
\hline \multirow{2}{*}{ Measure (N×m) } & \multicolumn{2}{|l|}{ Sham } & \multicolumn{2}{l|}{ Collar } & \multirow{2}{*}{$\boldsymbol{P}$} \\
\cline { 2 - 5 } & Mean & SD & Mean & SD & \\
\hline Hip extension & 137.31 & 48.09 & 136.27 & 50.98 & 0.793 \\
\hline Hip abduction & 20.34 & 16.90 & 19.70 & 14.58 & 0.781 \\
\hline Hip external rotation & 31.29 & 11.76 & 30.79 & 10.86 & 0.670 \\
\hline Knee extension & -136.53 & 37.89 & -134.56 & 37.60 & 0.609 \\
\hline Knee abduction & -25.90 & 15.59 & -22.66 & 12.62 & $0.045^{\text {a }}$ \\
\hline Knee internal rotation & 11.75 & 9.28 & 12.29 & 8.41 & 0.479 \\
\hline Ankle plantarflexion & 111.90 & 29.52 & 108.77 & 26.10 & 0.226 \\
\hline Ankle eversion & -18.39 & 9.57 & -18.02 & 8.55 & 0.761 \\
\hline
\end{tabular}

Abbreviation: SD, standard deviation.

andicates statistical significance $p<0.05$. 
Table 4 Comparison of mean and standard deviation dynamic stabilization scores between the Sham and Collar conditions

\begin{tabular}{|c|c|c|c|c|c|}
\hline \multirow[t]{2}{*}{ Measure } & \multicolumn{2}{|l|}{ Sham } & \multicolumn{2}{|l|}{ Collar } & \multirow[t]{2}{*}{$p$} \\
\hline & Mean & SD & Mean & SD & \\
\hline Right mean overall score & 3.5 & 1.5 & 5.7 & 9.0 & 0.341 \\
\hline Right mean anteroposterior score & 2.3 & 1.1 & 2.5 & 1.4 & 0.843 \\
\hline Right mean mediolateral score & 2.1 & 1.1 & 2.0 & 1.0 & 0.827 \\
\hline Right SD overall score & 1.9 & 1.1 & 2.0 & 1.0 & 0.915 \\
\hline Right SD anteroposterior score & 1.8 & 0.9 & 1.8 & 1.0 & 0.864 \\
\hline Right SD mediolateral score & 1.4 & 0.8 & 1.4 & 0.7 & 0.789 \\
\hline Left mean overall score & 3.7 & 1.5 & 3.2 & 1.3 & 0.402 \\
\hline Left mean anteroposterior score & 2.6 & 1.0 & 2.4 & 1.2 & 0.599 \\
\hline Left mean mediolateral score & 1.9 & 1.1 & 1.6 & 0.9 & 0.377 \\
\hline Left SD overall score & 1.9 & 1.0 & 1.7 & 0.7 & 0.538 \\
\hline Left SD anteroposterior score & 1.8 & 0.8 & 1.7 & 0.7 & 0.571 \\
\hline Left SD mediolateral score & 1.5 & 1.0 & 1.2 & 0.6 & 0.279 \\
\hline
\end{tabular}

Abbreviation: SD, standard deviation.

Table 5 Comparison of mean Y-Balance scores between the Sham and Collar conditions

\begin{tabular}{|c|c|c|c|c|c|}
\hline \multirow[t]{2}{*}{ Measure $(\mathrm{cm})$} & \multicolumn{2}{|l|}{ Sham } & \multicolumn{2}{|l|}{ Collar } & \multirow[t]{2}{*}{$p$} \\
\hline & Mean & SD & Mean & SD & \\
\hline Right Anterior Score & 57.8 & 4.5 & 56.1 & 5.6 & 0.317 \\
\hline $\begin{array}{l}\text { Right Posteromedial } \\
\text { Score }\end{array}$ & 87.6 & 7.0 & 88.4 & 8.8 & 0.831 \\
\hline $\begin{array}{l}\text { Right Posterolateral } \\
\text { Score }\end{array}$ & 89.8 & 13.6 & 86.0 & 8.6 & 0.286 \\
\hline Left Anterior Score & 56.8 & 5.4 & 56.3 & 4.9 & 0.767 \\
\hline $\begin{array}{l}\text { Left Posteromedial } \\
\text { Score }\end{array}$ & 93.9 & 9.1 & 93.9 & 7.1 & 0.984 \\
\hline Left Posterolateral Score & 81.1 & 7.5 & 83.1 & 10.6 & 0.554 \\
\hline
\end{tabular}

Abbreviation: SD, standard deviation.

Table 6 Comparison of mean peak knee flexion and extension torques between the Sham and Collar conditions

\begin{tabular}{|l|l|l|l|l|l|}
\hline Measure (N*m) & Sham & SD & Collar & SD & \multirow{2}{*}{$p$} \\
\cline { 2 - 2 } & Mean & & Mean & & \\
\hline Right knee extension & 84.1 & 25.7 & 85.5 & 26.4 & 0.906 \\
\hline Right knee flexion & 49.9 & 17.1 & 53.6 & 20.4 & 0.651 \\
\hline Left knee extension & 86.3 & 25.0 & 84.1 & 23.5 & 0.782 \\
\hline Left knee flexion & 50.8 & 14.9 & 53.9 & 19.1 & 0.686 \\
\hline $\begin{array}{l}\text { Maximum vertical } \\
\text { jump (in.) }\end{array}$ & 18.5 & 4.6 & 18.6 & 4.5 & 0.926 \\
\hline
\end{tabular}

Abbreviation: SD, standard deviation.

primary motor cortex, basal ganglia, and cerebellum-the regions responsible for complex motor control. Acceptability and tolerance of a device, such as the one tested in this investigation in an athletic population, are strongly dependent on these findings that wearing this device does not negatively affect an athlete's ability to perform on the field or court.

\section{Conclusion}

The current study intended to address the safety of the Collar while being worn by individuals performing athletic tasks. The investigators applied a rigorous array of metrics to elucidate any adverse findings that might develop while imparting jugular compression during a multitude of physiologic and neurologic challenges intended to mimic competitive sport. No negative findings could be found even under robust statistical analysis and under a prospective, blinded (sham) protocol. The effectiveness of the Collar to mitigate brain injury, for which it was originally designed, should be studied separately.

\section{Conflict of Interests}

None.

\section{Funding}

Q30 Innovations provided funding to Cincinnati Children's Hospital for the current investigation.

\section{References}

1 Smith DW, Bailes JE, Fisher JA, Robles J, Turner RC, Mills JD. Internal jugular vein compression mitigates traumatic axonal injury in a rat model by reducing the intracranial slosh effect. Neurosurgery 2012;70(03):740-746

2 Smith DW, Myer GD, Currie DW, Comstock RD, Clark JF, Bailes JE. Altitude modulates concussion incidence: implications for optimizing brain compliance to prevent brain injury in athletes. Orthop J Sports Med 2013;1(06):2325967113511588

3 Myer GD, Yuan W, Barber Foss KD, et al. The effects of external jugular compression applied during head impact exposure on longitudinal changes in brain neuroanatomical and neurophysiological biomarkers: a preliminary investigation. Front Neurol 2016;7:74 
4 Myer GD, Yuan W, Barber Foss KD, et al. Analysis of head impact exposure and brain microstructure response in a season-long application of a jugular vein compression collar: a prospective, neuroimaging investigation in American football. $\mathrm{Br} \mathrm{J}$ Sports Med 2016;50(20):1276-1285

5 Hatt A, Cheng S, Tan K, Sinkus R, Bilston LE. MR elastography can be used to measure brain stiffness changes as a result of altered cranial venous drainage during jugular compression. AJNR Am J Neuroradiol 2015;36(10):1971-1977

6 Hewett TE, Myer GD, Ford KR, et al. Biomechanical measures of neuromuscular control and valgus loading of the knee predict anterior cruciate ligament injury risk in female athletes: a prospective study. Am J Sports Med 2005;33(04):492-501

7 Ford KR, Myer GD, Hewett TE. Valgus knee motion during landing in high school female and male basketball players. Med Sci Sports Exerc 2003;35(10):1745-1750

8 Myer GD, Ford KR, Hewett TE. Rationale and clinical techniques for anterior cruciate ligament injury prevention among female athletes. J Athl Train 2004;39(04):352-364

9 Ruedl G, Webhofer M, Helle K, et al. Leg dominance is a risk factor for noncontact anterior cruciate ligament injuries in female recreational skiers. Am J Sports Med 2012;40(06):1269-1273
10 Paterno MV, Schmitt LC, Ford KR, et al. Biomechanical measures during landing and postural stability predict second anterior cruciate ligament injury after anterior cruciate ligament reconstruction and return to sport. Am J Sports Med 2010;38(10): 1968-1978

11 Myer GD, Ford KR, Barber Foss KD, Liu C, Nick TG, Hewett TE. The relationship of hamstrings and quadriceps strength to anterior cruciate ligament injury in female athletes. Clin J Sport Med 2009; 19(01):3-8

12 Plisky PJ, Rauh MJ, Kaminski TW, Underwood FB. Star Excursion Balance Test as a predictor of lower extremity injury in high school basketball players. J Orthop Sports Phys Ther 2006;36(12): 911-919

13 Zazulak BT, Hewett TE, Reeves NP, Goldberg B, Cholewicki J. The effects of core proprioception on knee injury: a prospective biomechanical-epidemiological study. Am J Sports Med 2007; 35(03):368-373

14 Hewett TE, Torg JS, Boden BP. Video analysis of trunk and knee motion during non-contact anterior cruciate ligament injury in female athletes: lateral trunk and knee abduction motion are combined components of the injury mechanism. Br J Sports Med 2009;43(06):417-422 